\title{
Laparoscopic Ultrasound-Guided Radiofrequency Thermal Ablation of Hepatic Tumors: A New Coaxial Approach
}

Radiofrequency (RF) in-situ tissue ablation has expanded the indications for curative therapy for nodular hepatocellular carcinoma (HCC) and liver metastasis from colon cancer. Due to rapid innovations, the balance between the risks and benefits of the various techniques has not yet been clarified in relation to the percutaneous, laparoscopic, and laparotomy approaches [1]. Published reports on laparoscopic ultrasound-guided radiofrequency (LUS-RF) tumor ablation have suggested potential benefits: reduced risk in difficult percutaneous approaches (hemoperitoneum, intestinal perforation, pleural complications), an opportunity for better staging [2] and simultaneous treatment of incidental nodules, the ability to switch to LUS-RF therapy after a previously planned surgical resection, and combination therapy in patients with multifocal disease. In LUS-RF, inser-

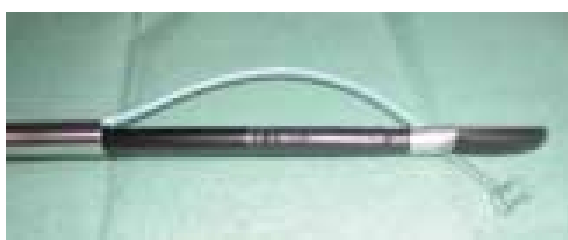

Figure 1 External view of the "armed" laparoscopic ultrasound probe, with the radiofrequency needle expanded into a spiral shape outside the operating channel.

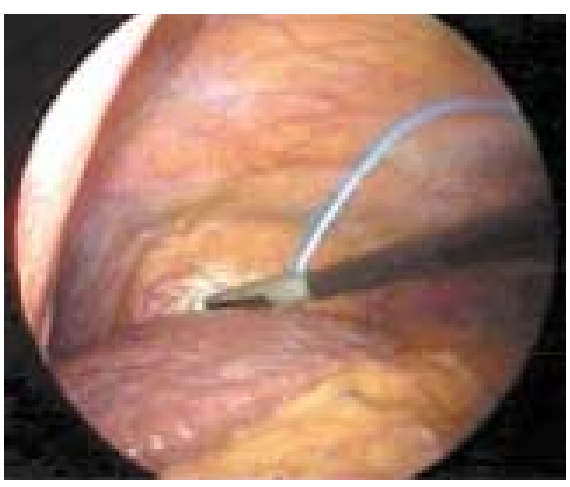

Figure 2 Laparoscopic view. The laparoscopic ultrasound probe is in contact with segment II of the left lobe of the liver. The blue catheter is "armed" with the radiofrequency needle. tion of the needle-electrode is usually carried out freehand through the abdominal or intercostal wall, and the needle tip is then directed under visualization on the laparoscopy monitor to the vicinity of the ultrasound transducer in contact with the liver surface. Correct placement of the needle in the lesion is assisted and monitored on the ultrasound screen. Access to some liver segments therefore requires mobilization of the liver itself, after resection of the right or falciform ligaments to allow the required direct laparoscopic visual control [3]. We present here a new fine-needle $(1-\mathrm{mm})$ instrument containing an expandable spiral electrode with a cylindrical shape, which is capable of ablating lesions up to $2.5 \mathrm{~cm}$ in diameter (Invatec, Italy). With this instrument, laparoscopy is carried out with the patient under conventional general anesthesia, and two ports are used - one for the laparoscope and one for the ultrasound probe. The LUS flexible probe (8666, B-K Medical, Denmark) is mounted on a biopsy system in which a 2-mm catheter is placed coaxially to the probe itself and screwed into the operating channel at the end of the transducer Figure 1. The probe enters the port under laparoscopic control, and upward movement allows straightening of the catheter angle with the operating channel. The fine-needle electrode is now pushed inside the catheter as far as the end of the probe's operating channel. This "armed" probe, with the

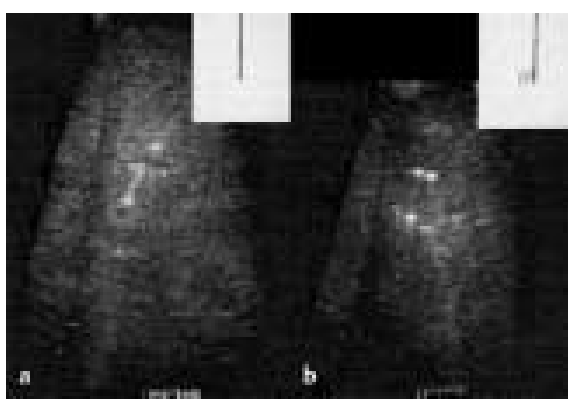

Figure 3 Ultrasound image and radiofrequency electrode. a The tip of the needle is inside the target liver nodule. b The spiral electrode expanded outside the needle, in the nodule. correct catheter curve, can be freely moved in contact with the liver surface, even without laparoscopic vision Figure 2. When the biopsy markers on the ultrasound monitor screen match the target nodule, the needle is pushed into the lesion and the spiral electrode is then expanded outside Figure 3. Two HCC nodules, $3 \mathrm{~cm}$ and $2 \mathrm{~cm}$ in size, were treated using this technique, as a percutaneous approach was not possible due to the site of the nodules in the upper right lobe in two patients with Child's grade B cirrhosis. Coaxial LUS-RF with the spiral electrode achieved $90 \%$ and $100 \%$ necrosis of the two nodules, respectively. This technique could became the method of choice in nodules in difficult sites or in small incidentally diagnosed nodules, for LUS staging of resectable liver tumors before laparotomy.

\section{A. Salmi ${ }^{1}$, F. Metelli ${ }^{2}$}

${ }^{1}$ Division of Gastroenterology, St. Orsola Fatebenefratelli Hospital, Brescia, Italy

${ }^{2}$ Division of Surgery, St. Orsola

Fatebenefratelli Hospital, Brescia, Italy

\section{References}

${ }^{1}$ Elias D, DeBaere T. La radiofréquence dans les tumeurs du foie. J Chir 2001; 38: $81-88$

${ }^{2}$ Catheline JM, Turner R, Champault G. Laparoscopic ultrasound of the liver. Eur J Ultrasound 2000; 12: 169-177

${ }^{3}$ Cuschieri A, Bracken J, Boni L. Initial experience with laparoscopic ultrasoundguided radiofrequency thermal ablation of hepatic tumours. Endoscopy 1999; 31: $318-321$

\section{Corresponding Author}

\section{A. Salmi, M.D \\ Ospedale S. Orsola Fatebenefratelli UO Gastroenterologia}

Via Vittorio Emanuele II 27 25100 Brescia, Italy

Fax: $\quad+39-030-2971835$

E-mail: asalmi@oh-fbf.it 